\title{
On the light emitting hot evolving black holes
}

\author{
B. Ramanuja Srinivas ${ }^{1}{ }^{*}$, P. Kalyani ${ }^{1}$, U.V.S. Seshavatharam ${ }^{2}$ \\ ${ }^{1}$ Assoc Prof, St Mary's Group of Institutions, Chebrolu-522212, Guntur Dist. AP, India \\ ${ }^{2}$ Honorary faculty, I-SERVE, Alakapuri, Hyderabad-50035, AP, India \\ *Corresponding author E-mail: brsmastan@gmail.com
}

\begin{abstract}
Along with the presently believed black holes that are expected to be formed by gravitational collapse of massive star, there may exist primordial very hot evolving black holes and their origin may be interlinked with the Planck mass. Extending this proposal, the current universe can be considered as a huge evolving black hole of radius equal to the current Hubble length and temperature equal to $2.725 \mathrm{~K}$. It can be suggested that 1) Vacuum is a great reservoir for matter and energy. 2) Planck mass can be considered as the primordial very hot baby black hole and can be called as the baby Planckion. 3) Baby Planckion will grow by absorbing the vacuum energy and thereby its geometric horizon also increases. As the baby Planckion is growing, its temperature and the tendency of vacuum energy absorption will decrease. 4) Very high temperature of the baby Planckion is the heat source for the evolving Planckion's decreasing temperature. 5) Growing Planckion can be considered as the seed and center of any galaxy. 6) Growing Planckion will permit the emission of radiation. 6) Current universe can be considered as a huge evolving Planckion with temperature $2.725 \mathrm{~K}$. In support this proposal, it is possible to guess that, in the past decreasing comic black whole temperature forced hydrogen atom to emit increased quantum of energy resulting in the observed redshift.
\end{abstract}

Keywords: Planck Scale, Planck ion, Evolving Black Holes, Black Hole Temperature, Emission of a Black Hole. CMBR Temperature, Evolving Black Hole Universe.

\section{Introduction}

Very recently S.W. Hawking modified his Black hole theory with "Apparent horizons" (S.W. Hawking 2014, p.3). This brought a serious confusion among the black hole physicists and whole science community. He says: "The absence of event horizons mean that there are no black holes - in the sense of regimes from which light can't escape to infinity. There are however apparent horizons which persist for a period of time. This suggests that black holes should be redefined as Meta stable bound states of the gravitational field". From this it can be understood that: There is no escape from a black hole in classical theory. Quantum theory, however, enables energy and information to escape from a black hole. He admits that, a full explanation of the process would require a theory that successfully merges gravity with the other fundamental forces of nature. But that is a goal that has eluded physicists for nearly a century. However 'the correct treatment'- 'remains a mystery'.

Abhas Mitra (Mitra A 2000) has shown that true black holes can never form. In his opinion the so-called black holes observed by astronomers are actually radiation pressure supported Eternally Collapsing Objects (ECOs). These balls of fire are so hot that even neutrons and protons melt there and whose outward radiation pressure balances the inward pull of gravity to arrest a catastrophic collapse before any Black Hole or 'singularity' would actually form.

Now the fundamental question to be answered is- Will any black hole exists without its event horizon? In author's opinion - the answer is "yes". By considering Hawking's view and Abhas Mitra's view, if one is willing to replace the "event horizon" with "geometric horizon", black holes can be considered as real 'very hot celestial quantum objects' with emission of electromagnetic energy under extreme gravitational attraction. Along with the presently believed black holes that are expected to be formed by gravitational collapse of massive star, there may exist primordial very hot evolving black holes and their origin may be interlinked with the Planck scale. These evolving black holes will grow by absorbing the hidden vacuum energy. As they grow their temperature will decrease. Clearly speaking their temperature is inversely proportional to the square root of their mass and the Planck mass. Whereas, with reference to Hawking black whole's temperature formula, collapsing black hole's temperature is inversely proportional to the mass of the black hole only. This is a very important point to be noted. Extending this proposal, the current universe can be considered as a huge evolving black hole of radius equal to the current Hubble length and temperature equal to the current cosmic microwave back ground temperature $2.725 \mathrm{~K}$. In reality one may see or may not see a black hole, but by considering the whole 'observable universe' as a huge primordial evolving black hole, many interesting things will come into review.

\subsection{To understand the growing geometric boundary of a growing black hole}

Generally any living or non-living object is being identified by its shape. In our daily life generally it is observed that any animal or fruit or human being (from birth to death) grows with closed boundaries (irregular shapes also can have a closed boundary). An apple grows like an apple. An elephant grows like an elephant. A plant grows like a plant. A human being grows like a human being. As their shape is being maintained continuously throughout their 
life time they won't change their respective identities. These are the observed biological facts. From these observed facts it can be suggested that "growth" or "expansion" can be possible with a closed boundary. Thinking that nature loves symmetry, in a heuristic approach in this paper authors assume that any black hole possesses a (growing) structural boundary by which its physical structure always seems to be identified as a (growing) black hole. Such type of boundary can be called as the growing geometric boundary of the growing black hole. Planck mass can be considered as the primordial very hot baby black hole. It can be considered as the seed of any growing black hole and can be called as the baby Planckion.

\subsection{Natural non-escaping of a freely falling body}

A freely falling body attains light speed when it reaches the black hole surface. At the same time it completely loses its shape and size due to black holes surface gravity and high temperature. The moment it reaches the light speed, (in a highly deformed state) it stats escaping from the black hole geometric horizon. Due to high surface gravity, its light speed escape velocity becomes zero within a short span. By any strange control mechanism if it is able to maintain its shape, size and light speed (against the black hole surface gravity and temperature), then certainly it will escape from the black hole geometric horizon.

\subsection{Natural escaping of photon}

Being a quantum mechanical object even though surface gravity is high photon will escape from the massive Planckion's geometric horizon. Clearly speaking during its escape from the massive Planckion's geometric horizon, photon may lose energy due to massive Planckion's surface gravity and show gravitational redshift but it will not lose its speed. Thus with increasing redshift photon will continue its journey until its energy becomes zero and redshift reaches infinity. For a photon moving towards the massive Planckion's horizon, its speed remaining constant it experiences gravitational blue shift and again speed remaining constant it leaves the massive Planckion's horizon by losing its acquired energy by gravitational redshift. Compared to the photon that originates from the black hole, photon that enters and leaves the black hole will make a long journey.

\section{The classical limits of force and power}

Without considering the current notion of black hole physics, Schwarzschild radius of black hole can be estimated with the characteristic limiting force of magnitude $\left(c^{4} / G\right)$. The outstanding problem in particle physics today is the inclusion of gravity in a single, unified quantum theory of all the fundamental interactions. Particle physicists have long suggested that the four observed fundamental forces of nature (the gravitational, electromagnetic, weak nuclear and strong nuclear forces) are separate, low energy manifestations of what was once a single force at times close to the Big Bang. It is postulated that as the universe expanded and cooled, this single force gradually broke down into the four separate interactions as observed today. However, unification theories that seek to unify the force of gravity with all the other forces (Theories of Everything) remain elusive, as the gravitational interaction lacks a quantum formulation.

To unify cosmology, quantum mechanics and the four observed fundamental cosmological interactions - certainly a 'unified force' is required. In this connection $\left(c^{4} / G\right)$ can be considered as the classical force limit. Similarly $\left(c^{5} / G\right)$ can be considered as the classical power limit. If it is true that $c$ and $G$ are fundamental physical constants in physics, then $\left(c^{4} / G\right)$ and $\left(c^{5} / G\right)$ can also be considered as fundamental compound physical constants. These classical limits are more powerful than the Uncertainty limit. Note that by considering the classical force limit $\left(c^{4} / G\right)$, the famous Planck mass can be obtained.

\subsection{Simple applications of $\left(c^{4} / G\right)$ can be stated as fol- lows}

a) Magnitude of force of attraction or repulsion between any two charged particles never crosses $\left(c^{4} / G\right)$.

b) Magnitude of gravitational force of attraction between any two massive bodies never crosses $\left(c^{4} / G\right)$.

c) Magnitude of mechanical force on a revolving/rotating body never crosses $\left(c^{4} / G\right)$.

d) Magnitude of electromagnetic force on a revolving body never $\operatorname{crosses}\left(c^{4} / G\right)$.

2.2. Simple applications of $\left(c^{5} / G\right)$ can be stated as follows

a) Mechanical power never crosses $\left(c^{5} / G\right)$

b) Electromagnetic power never crosses $\left(c^{5} / G\right)$

c) Thermal radiation power never crosses $\left(c^{5} / G\right)$

d) Gravitational radiation power never crosses $\left(c^{5} / G\right)$

\section{To derive the Planck mass}

So far no theoretical model proposed a derivation for the Planck mass. Two derive the Planck mass the following two conditions can be given a chance.

Assuming that gravitational force of attraction between two Planck particles of mass $\left(M_{P}\right)$ separated by a minimum distance $\left(\mathrm{r}_{\min }\right)$ be,

$\left[\frac{G M_{P} M_{P}}{r_{\text {min }}^{2}}\right] \equiv\left(\frac{c^{4}}{G}\right)$

With reference to wave mechanics, let

$2 \pi \cdot r_{\text {min }} \cong \lambda_{P}=\left[\frac{h}{c . M_{P}}\right]$

Here, $\lambda_{P}$ represents the wavelength associated with the Planck mass. With these two assumed conditions Planck mass can be obtained as follows.

$M_{P}=\sqrt{\frac{h c}{2 \pi G}} \cong \sqrt{\frac{\hbar c}{G}}$

\section{Schwarzschild radius of a black hole}

The 4 basic physical properties of a rotating black hole are its mass, size, angular velocity and temperature. Without going deep into the mathematics of black hole physics in the following sub- 
sections an attempt is made to understand and fit the black hole radius and temperature.

In all directions if a force of magnitude $\left(c^{4} / G\right)$ acts on the mass-energy content of the assumed celestial body it approaches a minimum radius of $\left(G M / c^{2}\right)$ in the following way. Origin of the force $\left(c^{4} / G\right)$ may be due to self-weight or internal attraction or external compression.

$$
R_{\text {min }} \cong \frac{M c^{2}}{\left(c^{4} / G\right)} \cong \frac{G M}{c^{2}}
$$

If no force (of zero magnitude) acts on the mass content $M$ of the assumed massive body, its radius becomes infinity.

With reference to the average magnitude of $\left(0, \frac{c^{4}}{G}\right) \cong \frac{c^{4}}{2 G}$, the presently believed Schwarzschild radius can be obtained as

$$
(R)_{\text {ave }} \cong \frac{M c^{2}}{\left(c^{4} / 2 G\right)} \cong \frac{2 G M}{c^{2}}
$$

This proposal is very simple and seems to be different from the existing concepts of General theory of relativity.

\section{Temperature of a growing planckion or evolving black hole}

At any time, the growing Planckion's thermal energy density can be estimated with the following assumption or conjecture. With this (classical) conjecture, Hawking's quantum mechanical black hole temperature formula (S.W. Hawking 1975) for Planck mass can be obtained. To prove it, it is must to measure the mass, size and temperature of any black hole. With reference to the current comic microwave back ground temperature and current Hubble length (David N Spergel et al.2013) it can be verified!

$a T_{t}^{4}\left(\frac{4 \pi}{3} R_{t}^{3}\right) \cong \frac{M_{t} c^{2}}{5760 \pi}$

Here, $\left(M_{t}, R_{t}, T_{t}\right)$ represent the instantaneous mass, radius and temperature of the growing black hole or growing Planckion respectively. With respect to the compound radiation constant $a \cong \frac{\pi^{2}}{15} \frac{k_{B}^{4}}{\hbar^{3} c^{3}}$ and the Planck mass $M_{P} \cong \sqrt{\frac{\hbar c}{G}}$, above relation takes the following simple form.

$T_{t}=\frac{\hbar c^{3}}{8 \pi k_{B} G \sqrt{M_{P} M_{t}}}$

Here, $M_{P}$ represents the baby Planckion mass. It is similar to the expression derived by S.W. Hawking for the black hole temperature with a change in the mass of the black hole. According Hawking, temperature of a black hole is given by the following famous relation.

$T_{B}=\frac{\hbar c^{3}}{8 \pi k_{B} G M_{B}}$

Here, $\left(M_{B}, T_{B}\right)$ represent the mass and temperature of the black hole respectively. Note that, so far Hawking's proposal is not verified and not confirmed by any of the advanced astrophysical observations or Large Hadron Collider experiments (Steven B. Giddings et al. 2002, F. Belgiorno et al. 2010). It is being believed based on the advanced quantum mechanical theoretical and mathematical formulations. Now, above relation can be re-expressed as follows.
$T_{t}=\sqrt{\frac{M_{t}}{M_{P}}} \cdot\left(\frac{\hbar c^{3}}{8 \pi k_{B} G M_{t}}\right)$

At the Planck scale,

$T_{P}=\frac{\hbar c^{3}}{8 \pi k_{B} G M_{P}}$

Here, $T_{P}$ represents the baby Planckion temperature. At Planck scale, proposed view of black hole temperature and Hawking's view of black hole temperature seems to be the same. Baby Planckion mass possesses a very high temperature and can be considered as a very hot fire ball.

\section{Understanding the origin of galactic black hole masses}

Now it can be suggested that, beginning from the Planck scale, high temperature Planckion is growing by absorbing the vacuum energy. During its evolution as it is absorbing the vacuum energy, its mass and size both will increase and hence its temperature will decrease. As its temperature decreases, its vacuum energy absorption capacity decreases and hence its growth rate decreases with increasing mass or decreasing temperature. During cosmic evolution, as time passes, Planckion will grow and its growth rate decreases with its decreasing temperature. Very high temperature of the baby Planckion is the only heat source for the evolving Planckion's decreasing temperature. Note that, there is no other internal mechanism for the assumed temperature of any evolving black hole or evolving Planckion. Proceeding further, growing Planckion can be considered as the seed and center of any galaxy. With reference to the Compton wavelength of nucleon and electron two semi stable massive Planckions can be estimated in the following.

Case-1: Nucleon and its Compton wave length

During cosmic evolution, as time passes at one particular instant, mass density of the growing Planckion approaches the nucleon and its Compton wavelength. Clearly speaking,

$\left[M_{t} c^{2}\left(\frac{4 \pi}{3} R_{t}^{3}\right)^{-1}\right] \Rightarrow m_{n} c^{2}\left(\frac{4 \pi}{3}\left(\frac{\hbar}{m_{n} c}\right)^{3}\right)^{-1}$

Where $m_{n}$ represents the mass of nucleon. If so the mass of the heavy Planckion can be obtained as follows.

$M_{t} \cong \sqrt{\frac{\hbar^{3} c^{3}}{8 G^{3} m_{n}^{4}}} \cong \frac{M_{P}^{3}}{\sqrt{8} m_{n}^{2}} \cong 1.3 \times 10^{30} \mathrm{~kg}$.

This can be compared with the current estimates of black hole masses and the Chandrasekhar mass limit $\cong 2.88 \times 10^{30} \mathrm{~kg}$ (S. Chandrasekhar 1931). From relation (7) its corresponding temperature can be estimated to be $7.32 \times 10^{11} \mathrm{~K}$. Note that this temperature is sufficient to heat the black hole's surrounding matter that is expected to emit X-rays.

\section{Case-2: Electron and its Compton wave length}

During cosmic evolution, as time passes at one particular instant, mass density of the growing Planckion approaches the electron and its Compton wavelength. Clearly speaking,

$\left[M_{t} c^{2}\left(\frac{4 \pi}{3} R_{t}^{3}\right)^{-1}\right] \Rightarrow m_{e} c^{2}\left(\frac{4 \pi}{3}\left(\frac{\hbar}{m_{e} c}\right)^{3}\right)^{-1}$

Where $m_{e}$ represents the mass of electron. If so the mass of the heavy Planckion can be obtained as follows.

$M_{t} \cong \sqrt{\frac{\hbar^{3} c^{3}}{8 G^{3} m_{e}^{4}}} \cong \frac{M_{P}^{3}}{\sqrt{8} m_{e}^{2}} \cong 4.38 \times 10^{36} \mathrm{~kg}$. 
This can be compared with the current assumed galactic black hole masses. From relation (7) its corresponding temperature can be estimated to be $4.0 \times 10^{8} \mathrm{~K}$.

\section{Understanding the origin of cosmic temper- ature and fitting the current Hubble con- stant}

If one is willing to consider the current universe as a huge Planckion of temperature 2.725 K (David N Spergel et al.2013), the currently believed Hubble length and hence the current Hubble constant can be fitted in the following way.

$$
\text { Let, } T_{0}=\sqrt{\frac{M_{t}}{M_{P}}} \cdot\left(\frac{\hbar c^{3}}{8 \pi k_{B} G \sqrt{M_{P} M_{0}}}\right) \cong 2.725 \mathrm{~K}
$$

Then,

$$
\begin{aligned}
\mathrm{M}_{0} & \cong \frac{\hbar^{2}{ }^{6}}{(8 \pi)^{2} k_{B}^{2} G^{2} T_{0}^{2} M_{P}} \cong \frac{M_{P}^{3} c^{4}}{(8 \pi)^{2} k_{B}^{2} T_{0}^{2}} \\
& \cong 9.31 \times 10^{52} \mathrm{~kg} .
\end{aligned}
$$

This can be considered as the current mass of the huge Planckion. Hence its radius can be estimated in the following way.

$$
R_{0} \cong \frac{2 G M_{0}}{c^{2}} \cong 1.382 \times 10^{26} \mathrm{~m} \text {. }
$$

If one is willing to consider this length as the current Hubble length, then current Hubble constant can be fitted as follows.

$R_{0} \cong \frac{2 G M_{0}}{c^{2}} \cong \frac{c}{H_{0}} \Rightarrow H_{0} \cong \frac{c}{R_{0}} \cong \frac{c^{3}}{2 G M_{0}}$

\section{$\cong 66.92 \mathrm{~km} / \mathrm{sec} / \mathrm{Mpc}$}

This obtained value is matching with the current estimates of the Hubble constant (David N Spergel et al.2013): $\{(67.80 \pm 0.77),(68.1 \pm 1.2)\} \mathrm{km} / \mathrm{sec} / \mathrm{Mpc}$. Based on this proposal, by this time the current universe must show a decelerating signal. At present with reference to $\left[\frac{d}{d t}\left(T_{0}\right)\right.$ and $\left.\frac{d}{d t}\left(H_{0}\right)\right]$ current 'true' cosmic rate of expansion can be understood. To confirm this proposal, it is necessary to reinterpret the Hubble's redshift. With reference to the concepts of standard cosmology, this new proposal seems to be simple in understanding and formulation but differs with the presently believed cosmic acceleration. At any time in the past, for the evolving cosmic black hole it can be shown that,

$\frac{T_{t}}{T_{0}} \cong \sqrt{\frac{H_{t}}{H_{0}}}$

\section{To understand and to reinterpret the galac- tic redshift}

The 3 great facts of cosmology were: galactic redshift, cosmic microwave background radiation temperature and super novae dimming. It is very important to note that, 1) If it is true that galaxy constitutes so many stars, each star constitutes so many hydrogen atoms and light is coming from the excited electron of galactic hydrogen atom, then considering redshift as an index of 'whole galaxy' receding may not be reasonable. 2) Increased redshifts and increased distances forced Edwin Hubble to propose the Hubble's law. Even then, merely by estimating galaxy distance and without measuring galaxy receding speed, one cannot verify its acceleration. Clearly speaking: two mistakes are being possible here. i) Assumed galaxy receding speed is not being measured and not being confirmed. ii) Without measuring and confirming the galaxy receding speed, how can one say and confirm that its re- ceding speed is increasing and accelerating? 3) If light is coming from the atoms of the gigantic galaxy, then instead of wavelength difference, in terms of 'quantum of energy' redshift can also be interpreted as a result of the cosmic temperature dependent atomic 'light emission mechanism'. 4) According to standard cosmology, since decoupling, the temperature of the CMBR temperature has dropped by a factor of roughly 1100 due to the expansion of the universe. As the universe expands, the $\mathrm{CMB}$ photons are redshifted, making the radiation's temperature inversely proportional to a parameter called the universe's scale factor. If $T_{t}$ is the temperature of the CMB and $z$ is the observed redshift, then $T_{t} \cong(1+z) 2.725 \mathrm{~K}$ where $(1+z)$ is known as the universal scale factor. Extending this concept, it can also be possible to guess that emitted quantum of energy is inversely proportional to the cosmic back ground temperature. If so aged and distant super novae dimming effect can also be considered as a result of high CBR temperature. In 1947 Hubble himself stated that (Hubble, E.P 1947, p.166-167): "We may predict with confidence that the 200 inch will tell us whether the red shifts must be accepted as evidence of a rapidly expanding universe, or attributed to some new principle in nature. Whatever may be the answer, the result may be welcomed as another major contribution to the exploration of the universe". Based on these points, the subject of standard cosmology can be reviewed at fundamental level. If one is willing to consider a 'light speed rotating cosmic black hole' its consequences seem to be very interesting (U. V. S. Seshavatharam et al. 2014).

Observed cosmic redshift can be reinterpreted in the following way. During cosmic evolution, at any time in the past, decreasing cosmic black hole's temperature forced hydrogen atom to emit increasing photon energy. Thus past light emitted from older galaxy's excited hydrogen atom will show redshift with reference to the current laboratory data. As cosmic time passes, in future, the absolute rate of cosmic expansion can be understood by observing the rate of increase in the magnitude of photon energy emitted from laboratory hydrogen atom. Aged super novae dimming may be due to the effect of high cosmic back ground temperature. Observed galactic redshift can be understood in the following way.

$$
\left.\begin{array}{l}
\frac{E_{0}}{E_{t}} \cong \frac{\lambda_{t}}{\lambda_{0}} \cong \frac{T_{t}}{T_{0}} \cong\left(z_{0}+1\right) \\
z_{0} \cong \frac{E_{0}-E_{t}}{E_{t}} \cong \frac{\lambda_{t}-\lambda_{0}}{\lambda_{0}} \cong \frac{T_{t}-T_{0}}{T_{0}}
\end{array}\right\}
$$

Here, $E_{t}$ is the energy of emitted photon from the galactic hydrogen atom and $E_{0}$ is the corresponding energy in the laboratory $\lambda_{t}$ Is the wave length of emitted and received photon from the galactic hydrogen atom and $\lambda_{0}$ is the corresponding wave length in the laboratory. $T_{t}$ Is the cosmic temperature at the time when the photon was emitted and $T_{0}$ is the current cosmic temperature and $z_{0}$ is the current redshift? This can be considered as the cosmic temperature dependent light emission mechanism in hydrogen atom.

At any time in the past - in support of the proposed cosmological red shift interpretation, in hydrogen atom, total energy of electron in $\mathrm{n}^{\text {th }}$ orbit can be expressed as follows.

$$
\begin{aligned}
\left(E_{\text {tot }}\right)_{t} & \cong-\left(\frac{T_{0}}{T_{t}}\right) \frac{e^{4} m_{e}}{32 \pi^{2} \varepsilon_{0}^{2} \hbar^{2} n^{2}} \\
& \cong-\sqrt{\frac{H_{0}}{H_{t}}} \cdot \frac{e^{4} m_{e}}{32 \pi^{2} \varepsilon_{0}^{2} \hbar^{2} n^{2}}
\end{aligned}
$$

Where $n=1,2,3, .$. From laboratory point of view, above concept can be understood in the following way. After some time in future,

$$
z_{f} \cong \frac{E_{f}-E_{0}}{E_{0}} \cong \frac{E_{f}}{E_{0}}-1
$$


Here, $E_{f}$ is the energy of photon emitted from laboratory hydrogen atom after some time in future. $E_{0}$ Is the energy of current photon emitted from laboratory hydrogen atom? $z_{f}$ Is the redshift of laboratory hydrogen atom after some time in future? In future within the scope of experimental accuracy of laboratory hydrogen atoms redshift $\left[d\left(z_{f}\right) / d t\right]$ can be considered as a true index of absolute rate of cosmic expansion. It can be understood from table-1 in the following way.

Table 1: To Understand the True Nature of Cosmic Expansion

\begin{tabular}{|c|c|c|}
\hline & Nature of change & Nature of cosmic expansion \\
\hline & Increasing & Acceleration \\
\hline$d(z f)$ & Constant & Uniform rate \\
\hline$d t$ & $\begin{array}{l}\text { Decreasing } \\
\text { Zero }\end{array}$ & $\begin{array}{l}\text { Deceleration } \\
\text { Zero }\end{array}$ \\
\hline
\end{tabular}

Note that, modern cosmologists believe that the rate of the change of the Hubble constant describes how fast/slow the Hubble constant changes over time and this rate does not tell if the Universe is currently expanding. This logic seems to be misleading. In authors opinion, if magnitude of past Hubble's constant was higher than the current magnitude then magnitude of past $\left(c / H_{t}\right)$ will be smaller than the current Hubble length $\left(c / H_{0}\right)$. If so rate of the decrease of the Hubble constant can be considered as a true index of rate of increase in Hubble length and thus with reference to Hubble length, rate of the decrease of the Hubble constant can be considered as a true index of cosmic rate of expansion. Proceeding further - in future, certainly with reference to current Hubble's constant, $d\left(c / H_{0}\right) / d t$ gives the true cosmic rate of expansion. Same logic can be applied to cosmic back ground temperature also. Clearly speaking $d\left(T_{0}\right) / d t$ gives the true cosmic rate of expansion. To understand the ground reality, sensitivity and accuracy of current methods of estimating the magnitudes of $\left(H_{0}\right.$ and $\left.T_{0}\right)$ must be improved.

\section{Discussion and conclusion}

Although quantum theory of gravity is needed in order to merge general relativity with the principles of quantum mechanics, difficulties arise when one attempts to apply the usual prescriptions of quantum field theory to the force of gravity. Out of all currently, there is still no complete and consistent quantum theory of gravity (Martin Bojowald 2012), and the candidate models still need to overcome major formal and conceptual problems. There are a number of other approaches to quantum gravity. The approaches differ depending on which features of general relativity and quantum theory are accepted unchanged, and which features are modified. One of the difficulties of quantum gravity is that quantum gravitational effects are only expected to become apparent near the Planck scale than what is currently accessible at high energy particle accelerators. As a result, current quantum gravity models seem to be pure theoretical models. It is accepted that a complete theory of the evolution of the universe will not be possible until the development of a successful quantum theory of gravity. Combining quantum mechanics and general theory of relativity is a must and needs conceptual fine tuning. In this context from the above concepts, logics, results and data fitting, it can be concluded that,

1) Along with presently the believed black holes that are expected to be formed by gravitational collapse of massive star, there may exist primordial evolving black holes.

2) Planck mass can be considered as the basic mass of any black hole and can be called as the baby Planckion. Very high temperature of the baby Planckion is the only heat source for the evolving black holes or evolving Planckion's decreasing temperature.
3) Evolving black hole or evolving Planckion temperature is inversely proportional to the square root of the baby Planckion mass and its evolving mass.

4) Any evolving black hole or evolving Planckion can emit electromagnetic radiation.

5) Current universe may be a growing huge Planckion of temperature $2.725 \mathrm{~K}$

6) Cosmic redshift can be reviewed as explained above in section-8.

\section{Acknowledgements}

All the authors honor the great scientific insight of S.W. Hawking and Abhas Mitra for their valuable contributions in black hole physics. The third author is indebted to professor K. V. Krishna Murthy, Chairman, Institute of Scientific Research on Vedas (ISERVE), Hyderabad, India and Shri K. V. R. S. Murthy, former scientist IICT (CSIR) Govt. of India, Director, Research and Development, I-SERVE, for their valuable guidance and great support in developing this subject.

\section{References}

[1] Hawking SW. arXiv: 1401.5761v1 (2014).

[2] Mitra A. Foundations of Physics Letters.13:543-579. (2000) http://dx.doi.org/10.1023/A:1007810414531.

[3] Hawking SW. Commun. Math. Phys.43:199-220. (1975) http://dx.doi.org/10.1007/BF02345020.

[4] David N. Spergel, Raphael Flauger and Renne Hlozek. Arxiv: $1312.3313 \mathrm{v} 1$.

[5] Steven B. Giddings and Scott Thomas. Phys.Rev. D65 056010 (2002) http://dx.doi.org/10.1103/PhysRevD.65.056010.

[6] Belgiorno, S. L. Cacciatori, M. Clerici, V. Gorini, G. Ortenzi, L. Rizzi, E. Rubino, V. G. Sala, and D. Faccio . Phys. Rev. Lett. 105, 203901 (2010) http://dx.doi.org/10.1103/PhysRevLett.105.203901.

[7] S. Chandrasekhar, Philosophical Magazine (7th series) 11 p.592-596 (1931).

[8] Hubble, E.P, PASP, 59, pp153-167, http://dx.doi.org/10.1086/125931.

[9] U. V. S. Seshavatharam, S. Lakshminarayana. Prespacetime Journal, Vol 5, Issue 7, pp.641-657, (2014).

[10]Martin Bojowald, Class. Quantum Grav. 29, 213001 (2012) http://dx.doi.org/10.1088/0264-9381/29/21/213001. 